Forthcoming in V. Petkov (ed.) (2010) Space, Time, and Spacetime - Physical and Philosophical Implications of Minkowski's Unification of Space and Time (Springer, Berlin, Heidelberg, New York)

\title{
Structural explanations in Minkowski spacetime: which account of models?
}

\author{
Mauro Dorato (University of Rome 3) \\ dorato@uniroma3.it \\ Laura Felline (University of Cagliari) \\ felline@uniroma3.it
}

\begin{abstract}
In this paper we argue that structural explanations are an effective way of explaining wellknown relativistic phenomena like length contraction and time dilation, and then try to understand how this can be possible by looking at the literature on scientific models. In particular, we ask whether and how a model like that provided by Minkowski spacetime can be said to represent the physical world, in such a way that it can successfully explain physical phenomena structurally. We conclude by claiming that a partial isomorphic approach to scientific representation can supply an answer only if supplemented by a robust injection of pragmatic factors.
\end{abstract}

In this paper we defend the thesis that structural explanations are an effective way of explaining well-known relativistic phenomena like length contraction and time dilation, and then try to understand how this can be possible by looking at the literature on scientific models. In particular, we ask whether and how Minkowski spacetime's model can be said to represent the physical world, in such a way that it can successfully explain physical phenomena structurally. In the first section, we try to briefly justify the above thesis by providing a brief sketch of structural explanations as they are used in Minkowski spacetime, in contrast to attempts at explaining the relativistic phenomena dynamically (Brown 2005). In the second section we offer a brief survey of the state of the art in the debate between the "representationalist" and the pragmatic conception of models, with particular attention to the inferentialist conception proposed by Suárez (1999, 2003, 2004). In the third section we argue that, in order both to solve some problems within Suárez's inferentialist approach and to account in a consistent way for the use that cognitive agents make of models, it is necessary to assume some kind of partial isomorphism between the mathematical model and the physical target. Our conclusion - the validity of which is here tested only in the specific case of structural explanations in Minkowski spacetime - makes the opposition between 
the pragmatic and the semantic view look much more apparent than real, and in fact proposes a reconciliation between the two points of view already defended with a different emphasis by Debs and Redhead (2007).

\section{Contractions, dilation and structural explanations}

Since the publication of Einstein's original paper on special relativity (SR), phenomena like rod contractions and clocks retardations have attracted the attention of philosophers. One of the key questions that has been raised by these phenomena from the very beginning was: are they real?

Of course the answer to a question like this depends on what one means by the metaphysically appealing but philosophically treacherous adjective "real" in our context. If "real" means "measurable", then the answer ought to be an uncontroversial "YES" written in capital letters, as every experimental physicist working at Fermi Lab or at the LHC in Geneva could guarantee. If "real" means "invariantly true", then the answer should also be yes, written in small letters though, considering the (italicized) relativization involved in the following claim: for all possible inertial observers, it seems true to say that, «relative to observer $O$, the rod contracted a certain amount $x$ in the direction of motion». However, if "real" means "dynamical", the vast majority of physicists and philosophers would answer the above question with a "NO", again written in capital letters. We don't need forces to account for the relativistic phenomena of contractions and dilations: after all, can't we explain such effects as, respectively, cross sections of four dimensionally conceived rods and projections of four-dimensionally conceived clocks onto different, arbitrarily chosen inertial frames of Minkowski spacetime? (see Figures below) ${ }^{1}$
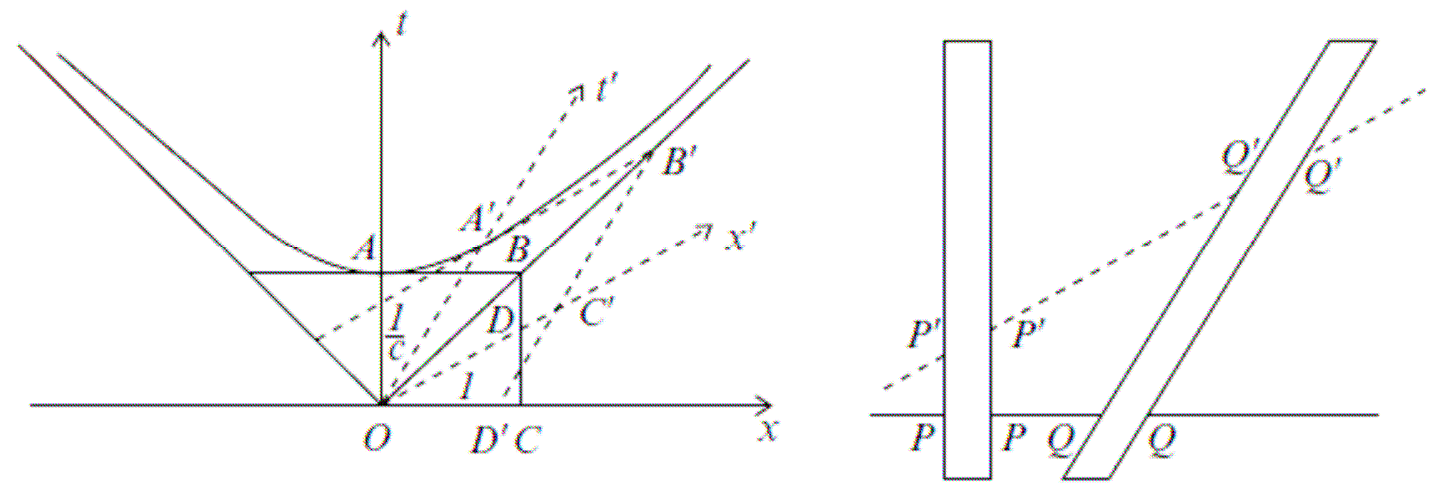

${ }^{1}$ Figure 1 is taken from (Minkowski 1908). Figure 2 is taken from (Petkov 2009), p. 86. 
Fig.1 Length contraction

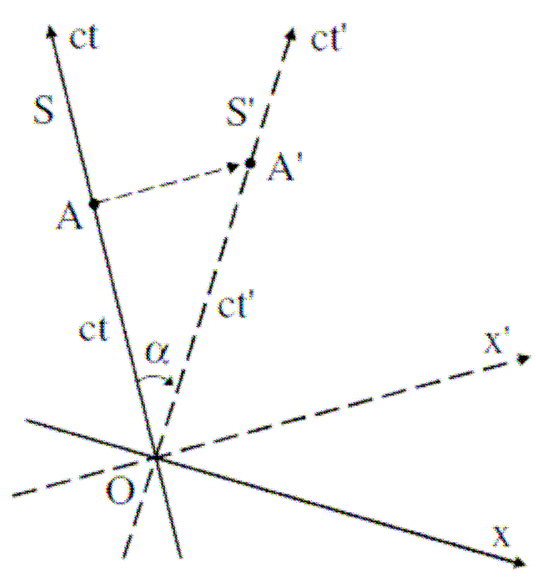

$$
\mid
$$

Fig.2 Time dilation

That is, we would add, we can explain such phenomena via structural explanations, based upon the geometrical features of Minkowski spacetime.

What are, however, structural explanations? A minimal definition of structural explanations was briefly provided by Rob Clifton:

We explain some feature B of the physical world by displaying a mathematical model of part of the world and demonstrating that there is a feature A of the model that corresponds to B. (Clifton 1998, p. 7, our emphasis)

The key problem raised by this brief quotation is of course what we should mean by "correspond"; a verb that calls into play the general problem of how mathematical models refer to the physical world, one of the main questions that this paper tries to tackle. Let us say at the outset that, in our understanding of structural explanations, their essential feature lies in the fact that their

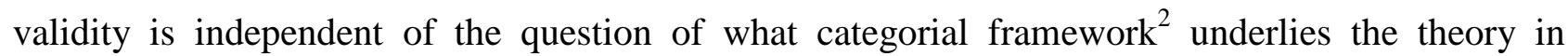
question, a thesis that typically allows one to neglect attempts at explaining phenomena by invoking causal or mechanistic models.

While the idea of structural explanation has mostly been developed by having quantum mechanics in mind (Dorato and Felline, forthcoming), also SR and the structure of Minkowski spacetime have already been regarded as a template of a theory providing structural explanations:

Suppose we were asked to explain why one particular velocity (in fact the speed of light) is invariant across the set of inertial frames. [...] [The Lorentzian] causal explanation is now seen as seriously misleading; a much better answer would involve sketching the models of space-time which special relativity provides and showing that in these models, for a certain family of pairs of events, not only is their spatial

\footnotetext{
${ }^{2}$ A categorial framework is the set of fundamental metaphysical assumptions about what sorts of entities and what sorts of processes lie within a theory's domain (Hughes 1989b).
} 
separation $\mathrm{x}$ proportional to their temporal separation $\mathrm{t}$, but the quantity $\mathrm{x} / \mathrm{t}$ is invariant across admissible (that is, inertial) coordinate systems; further, for all such pairs, $x / t$ always has the same value. This answer makes no appeal to causality; rather it points out structural features of the models that special relativity provides. It is, in fact, an example of a structural explanation (Hughes 1989b, pp. 256-257)

The following example will show in what sense structural explanations of physical phenomena in Clifton's sense can avoid any appeal to causality or forces. Suppose that we want to understand why it is the case that clocks in relative motion measure a time that is dilated with respect to the time measured by clocks at rest in the chosen inertial frame. The typical explanation that is provided in most textbooks is repeated from Feynman's lectures (Feynman, Leighton, Sands, 1963 I vol., 15-6). Take a light ray that goes up and down between two mirrors (figure on the left, below). Each round trip of the light ray (supposing it is originally emitted from the bottom) gives us one beat of our clock. But in the moving frame, which is endowed with the same kind of light clock, the observer at rest will observe that the light ray originating from $\mathrm{A}$ is reflected at B and then comes back at the bottom mirror at point C. (see Figure 3) Since the light ray must zigzag along a longer path, the measured time will be longer. The explanation in question is obviously geometrical/algebraic, and therefore structural in the sense specified by Clifton. It is geometrical because in order to show the dependence of the dilation on the relative velocity, it relies on Pythagoras' theorem: if the hypotenuse in Figure 3 has a length $\mathrm{c}$, and the moving clock travels a distance $2 u$ from emission to reception of the pulse, the height of the triangle is $\left(c^{2}-u^{2}\right)^{1 / 2}$ which is obviously smaller than $c$, so that $2\left(c^{2}-u^{2}\right)^{1 / 2}<2 c$.
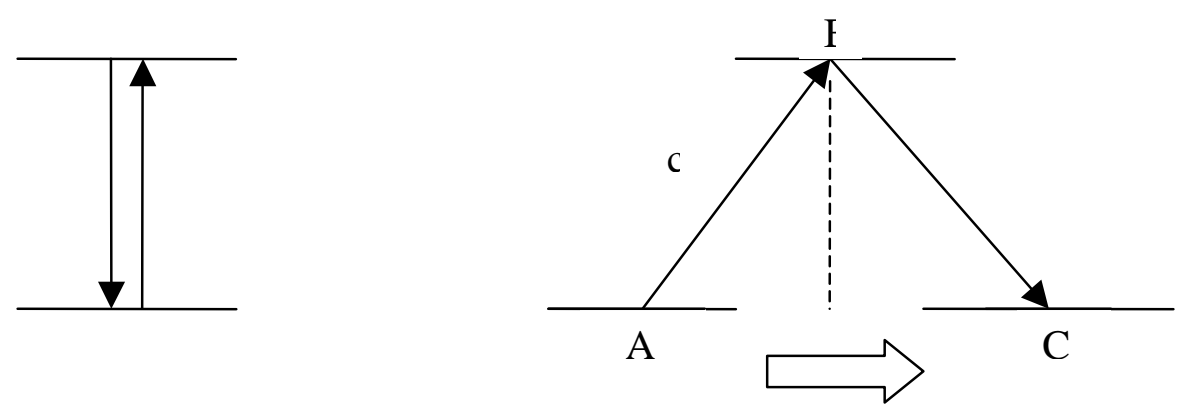

Fig. 3

Despite examples of this kind, Harvey Brown (2005) and some co-workers (especially Dr. Oliver Pooley, a former, brilliant student of his (see Brown and Pooley 2006)) have instead been arguing for some years that length contractions and clock dilations, so far often regarded as purely kinematical effects, need a dynamical, presumably quantum explanation, in terms of Lorentz covariant laws, an explanation that does not require a privileged inertial frame. 
The following brief (and admittedly incomplete) criticism of Brown's dynamical proposal (but see Dorato 2007 and Norton 2008) will however serve to illustrate in what sense structural explanations of length contraction rely on the structure of Minkowski spacetime, and as such do not presuppose dynamical effects. SR tells us that the amount of contraction of a body depends on the arbitrary choice of the measuring frame, and therefore on the relative velocity between the two inertial frames. If this is agreed upon, it is not clear why we should grant the deformation a dynamic significance, rather than a simple geometrical/structural significance. If relative to frame $f$ the contraction $\mathrm{F}$ of object $\mathrm{O}$ is $\mathrm{F}(f)$, relative to frame $\mathrm{f}^{\prime}$ is $\mathrm{F}\left(f^{\prime}\right)$, relative to $f^{\prime}$ ' is $\mathrm{F}\left(f^{\prime}\right.$ ') and so on, the implication that there is no intrinsic shape of the body $\mathrm{O}$ is quite natural, since length or shape in the special theory of relativity are non-invariant notions.

One could even be tempted to conclude that there is no fact to be explained. However, to the extent that relativistic phenomena do need an explanation, as we believe it is actually the case, ${ }^{3}$ structural explanations do suffice, in virtue of the following geometric and topological aspect of Minkowski spacetime. If we conceive spacetime and the physical world four-dimensionally, as recommended by Minkowski in his original paper (1908), we should conceive four-dimensionally also physical objects. Such four-dimensionality ought to be regarded as one of their key objective features, derived by the fact that we model them as "inhabitants" of Minkowski spacetime. ${ }^{4}$

The main fact to rely upon at this point is that fourdimensional entities can be "sliced" in different ways: according to the frame of reference that we happen to choose, we obtain a different spatial section of a fourdimensional entity, in the same sense in which, by slicing a fourdimensionally conceived electromagnetic field, we obtain different but separate electrical and magnetic fields. The geometrical aspect provided by the "slicing" (a cross-section) is what makes the explanation of length-contraction mathematical, and therefore, in Clifton's sense, structural: we are simply locating length contraction (the phenomena to be explained) in the mathematical model of Minkowski spacetime (the explanans). Notice that causation, mechanical models or dynamical forces are never called into play and they seem to be wholly superfluous.

Nevertheless, in defence of his claim of the necessity of a dynamical understanding of special relativity, Brown (2005, Brown and Pooley (2006), and Brown and Timpson (2006) have often appealed to the distinction between principle and constructive theories (Einstein 1919), and to the fact that SR, in Einstein's own opinion, is to be conceived as a principle theory. For the sake of brevity, Brown's argument can be summarized by the following two premises:

\footnotetext{
${ }^{3}$ Recall that there is a relational and objective matter of fact for all observers about the contraction of a ruler relative to an inertial wordline $O$ (see above).

${ }^{4}$ For the importance of questions of dimensionality in Minkowski spacetime, see Petkov (2007). For a defense of a fourdimensional metaphysics, which here we take for granted, see Sider (2003).
} 

spacetime cannot be regarded as explanations typical of constructive theories;

It then follows that, according to Brown, and contrary to what we argued so far, we still lack a genuine understanding of the phenomenon of length contraction or time dilation. A thorough understanding of the latter phenomena could only be provided by a 'constructive theory' in Brown's (controversial, from our point of view) sense. According to Einstein, a constructive theory is a theory that, like statistical mechanics, is capable of constructing, or giving a deeper account of, physical phenomena - phenomena that the principle theory instead constrains only via very general empirical principles which do not depend on hidden levels of description. ${ }^{5}$

In arguing for premise 2), Brown often relies on Yuri Balashov and Michael Janssen' (2003) characterization of the different way in which explanations are provided in principle theories and constructive theories respectively. This reliance, however, creates two sorts of difficulties, which here can only be sketched (but see Felline (forthcoming)). The first difficulty originates from the fact that Brown uses Balashov and Janssen' characterization in order to claim that principle theories typically provide Deductive-Nomological (DN) explanations, while constructive theories typically rely on model-based explanations. However, this claim, as some others based on this characterization, is unwarranted: as we hope to have shown above by illustrating the role of structural explanations in Minkowski spacetime, also principle theories are capable of providing perfectly acceptable model-based explanations, which (explicitly at least) do not mention any physical laws in their premises. To the extent that principle theories rely on structural explanations, it is not true that the latter are only based on DN explanations. Clearly, the sense in which structural explanations rely on "models" is certainly different from the sense in which "model" is used, say, for referring to the billiard-ball model typical of the kinetic theory of gases, the standard example of a constructive theory.

The second problem is that Brown misunderstands the way structural explanations function in the context of SR, by saddling them with an implausible causal type of substantivalism associated to Minkowski spacetime, a (dirty-water) substantivalism that he himself correctly rejects, unfortunately together with the baby (structural explanations) (Brown and Pooley 2006). In order to understand the origins of this unfair characterization of structural explanations, it is important to

\footnotetext{
5 "One can distinguish various kinds of theories in physics. Most of them are constructive. These seek to construct a model of the more complex phenomena out of a relatively simple formalism taken as a basis. Thus the kinetic theory of gases seeks to reduce mechanical, thermal, and diffusional processes to the movements of molecules-i.e., to construct them out of the hypothesis of molecular motion. When one says that we have succeeded in understanding a group of natural processes, one always means that a constructive theory has been found that comprehends the relevant processes" (Einstein 1919, transl. by Don Howard). It is possibly not irrelevant to remark that none so far has been able to provide any such constructive theory, neither for SR nor for GR.
} 
keep in mind his premise 2). Since according to him only constructive theories provide genuine explanations, and since only such explanations rely on "models", Brown is led to think that also geometrical explanations, if genuine, must function like the billiard-balls model of the kinetic theory. In order words, he is led to presuppose that since the representation provided by the model used in constructive explanations typically include a strong form of ontological commitment toward the target entities and processes, the same must hold for structural explanations within Minkowski spacetime.

A constructive model of spacetime conceived in this ontic sense would then represent spacetime as a substance which exists independently of, and acts on, things, events and processes immersed in it. A principle theory explanation would instead not be ontically committed at all. In support of the fact that our reading of Brown's approach to "model" is plausible, consider how well it fits with his (misleading) understanding of structural explanations, within what he calls the 'orthodox' view of SR - i.e., the 'constructive version' of the geometrical explanations provided by $S R$ and illustrated above in a non-causal, non-metaphysical way. According to Brown, in what he regards as the 'orthodox', geometrical explanation of the kinematic behaviour of bodies, Minkowski spacetime ought to play a causal role! But since, as Brown correctly notes, Minkowski spacetime cannot have the function of 'shaping' rods by causing in this way the Lorentz contractions, the unwarranted conclusion is that structural explanations cannot be effective. However, why saddling structural explanations with this causal-metaphysical baggage, when their main purpose is to do away with unwanted metaphysical assumptions? ${ }^{6}$ Thinking that in a physical theory like SR a geometrical/structural explanation can work only if it does so causally is reducing ad absurdum this view.

Here we will not further discuss Brown's arguments against the success of structural explanations in SR. For the aim of this paper, it is important to have shown how a poor understanding of the relation between mathematical models and the physical world may prevent philosophical progress and cause misunderstandings. In the next section we will therefore briefly present the debate on the nature of models, in order to see how one should properly understand the effectiveness of the geometrical explanations provided by the structure of Minkowski spacetime. How should we explain such explanations, given that the universally agreed-upon background dependence (or causal inertness) of Minkowski spacetime makes Brown's causal reading rather implausible? ${ }^{7}$

\footnotetext{
${ }^{6}$ For another improper, metaphysical use of Minkowski spacetime, see Dorato 2006.

${ }^{7}$ Given that also the metric field is causally inert even though it is surely correlated to matter (a causal reading of the metric field is very controversial to say the least), how could one regard a causal reading of Minkowski spacetime as plausible?
} 


\section{The debate on models: the state of the art}

The Semantic View (SV) has until very recently dominated the discussions in the theory of scientific models ${ }^{8}$. According to this view, a scientific model is a set-theoretic structure

$$
S=\langle U, O, R\rangle
$$

i.e. an abstract triple consisting of (i) a non-empty set $U$ of individuals called the domain of the structure $S$, (ii) an indexed set $O$ of operations on $U$ (which may be empty), and (iii) a non-empty indexed set $R$ of relations on $U$. Within the SV, the relation between a model and its target is traditionally defined as a dyadic relation of isomorphism or "embedding a physical theory in a mathematical structure" (French 1999, p. 188), or as a weaker relation of similarity (Giere 1988). However, both these relations have been found problematic and other morphisms have been put forward, like a relation of partial isomorphism (French and Ladyman 1999).

The plausibility of such accounts as an explication of the concept of scientific representation has recently been challenged (see Suárez, 2003 and Frigg 2006). According to Frigg, for instance, while it can be considered a requirement for the accuracy of some kind (or, with Frigg's term, style) of scientific representations, isomorphisms (or other dyadic relations of morphism) are in general not sufficient to account for the way cognitive agents (scientists) utilize models in order to perform "surrogative reasoning" about the target (physical) system. In other words, to argue that a model $M$ represents a target $S$ iff $M$ is isomorphic to $S$ is not sufficient to explain the complex relations holding between representation and the different uses that are made of models. For instance, the relation of isomorphism is symmetric, while the relation of representation clearly isn't. Isomorphisms can hold only between two abstract, already mathematized structures, not between an abstract structure and a physical target, so that we must focus on data which have already been mathematised (Suppes'models of data (1962)); furthermore, the same abstract model can be multiply realized, so that there is a problem of under-determination of reference, accompanied by the fact that the same target can exemplify many different structures. Finally isomorphisms, unless partial, seems incapable of explaining cases of misrepresentation (think of gas molecules represented as billiard balls: of course, they are not literally "balls").

In order to overcome all of these problems, a more pragmatic approach has been proposed, one that focuses more on the use that cognitive agents make of models. In particular, Mauricio Suárez proposes a deflationary approach to scientific representation, according to which: "[r]epresentation is not the kind of notion that requires, or admits such [universal necessary and

\footnotetext{
${ }^{8}$ See Suppes 1967, Suppe 1977, van Fraassen 1980, Giere 1988.
} 
sufficient] conditions. [...] finding necessary conditions will certainly be good enough.” (Suárez 2006, p.771, see also van Fraassen 2008).

Suárez correctly claims that if a theory is meant to account for our deep-grounded intuitions about scientific representations, it must also account for the fact that a scientific representation is not just the product of an arbitrary convention between agents. Consequently, there must be something in the model $M$ that makes it the case that a cognitive agent can legitimately use $M$ to perform surrogative reasoning about the target. But here, let us note, some potential room is made for a more conciliatory view between the model-theoretic and the pragmatic camp. Scientific models, we are told, do more than merely denote an object, as they allow us to draw relevant conclusions about their target: in other words, they are informative about it. Suárez claims that it is in virtue of their informativity that scientific models are objective. This word is clearly ambiguous between "intersubjectively shared" and "representing properties of the target existing independently of the model". Given his anti-representationalist approach, one expects Suárez to opt decisively for the former, merely epistemic alternative, while rejecting the latter, more ontological version, which would take us back toward some sort of isomorphic view of representation.

Suárez's proposal is that it is exactly the capacity to allow for "surrogative reasoning" that determines the objectivity of scientific models. Scientific representation is therefore characterized by the following criterion:

[inf]: A represents B only if (i) the representational force of A points towards B, and (ii) A allows

competent and informed agents to draw specific inferences regarding B. (ibid., p. 771)

The representational force of a source (or, more simply, the force of a model) is "the capacity of a source to lead a competent and informed user to a consideration of the target", in virtue of "a relational and contextual property of the source, fixed and maintained in part by the intended representational uses of the source on the part of agents": (ibid., p. 768, our emphasis).

Which relational and contextual property of the source is Suárez talking about? The answer is that he cannot be more specific about it, since more specificity would push him toward admitting the possibility of some sort of well-defined relation always existing between model and target, and he denies exactly this point. And yet, it is “[inf]'s part (ii) that has the important function of contributing to the objectivity that characterises scientific representation. In contrast to part (i), the above mentioned relational property in no way depends on an agent's existence or cognitive activity. It requires the model $A$ to have the internal structure that allows informed agents to correctly draw inferences about the physical target $B$, but it does not require that there be any agent who actually does so." (ibid., p.774). This is still very vague, as the reader will recognize, but the idea is that the "internal structure of the model" is not fixed by the context of inquiry, and it is this 
non-contextuality which, according to our author, guarantees the objectivity or non-arbitrariness of the model.

Suárez explains how the concepts of informativity and objectivity are related with an example. Consider a peace of paper and two pens writing on it, and stipulate that they represent respectively the sea and two ships sailing on it. Compare then this representation with the opposite one, in which the paper represents the ships and the pens represent the sea. Suárez claims that "the ships-on-sea system is more "objectively" characterised by the first denotational arrangement than by the second" and with this he means that the second representation "is certainly less informative, since the relative movements of pens and paper can not allow us, for instance, to infer the possibility that the two ships may crash" (p. 8).

To see why Suárez's proposal cannot be satisfactory, consider the following situation. If it is true that, with the referential conventions: ships=paper, pens=sea, one cannot draw the conclusion that the two ships may crash, it must be admitted that in this non-standard representation one can draw other interesting conclusions. One can for instance conclude (under the assumption that the paper is rigid) that whatever movements the ships can perform, they will always remain at the same distance one from the other, or that if you burn one of them also the other will burn. These conclusions are obviously false, since, after all, the piece of paper is unique, and the example has it that there are two separate ships; but if some degree of faithfulness and accuracy of the representation to the physical reality is always irrelevant for objectivity, then one cannot argue that one representation is more objective than the other: the two are on a par. One can at most claim that one representation allows to draw more inferences than the other.

It then seems that we cannot discriminate between objective and non-objective representations without providing some more specific characterization of the kind of relation that must hold between target and model. The above example of the pens, which might simply be badly chosen, suggests, however, something relevant to our main purpose: it is the good degree of "faithfulness" to the world of one of two representations that allows a more accurate and reliable surrogative reasoning; it is this accuracy that, in its turn, measures the objectivity of a model. After all, a very good reason for more or less informativity or inferential power is given by the fact that one representation captures key features of the world (target) much better than the other.

Suárez rejects this account from the very beginning. He does so because, he argues, if objectivity were defined in terms of "truthfulness" or accuracy, it would be very difficult (if not impossible) to account for cases of misinterpretation, inaccuracy, idealizations, etc. ${ }^{9}$ However, the notion of surrogative reasoning must be obviously further characterized: the requirement that a user

\footnotetext{
${ }^{9}$ Batterman (2010) argues that asymptotic behaviour cannot be captured by any kind of isomorphic relation.
} 
can draw inferences about the target is too weak to grasp the objectivity of representation, since one can draw irrelevant, or even wrong inferences; and if one has to restrict one's attention to successful inferences, one might be in need of an explanation of this success that is not circular, i.e., that is not given in terms of informativity.

In a word, the problem with Suárez's account is that, remaining as it does wholly on the epistemic terrain, there is no possibility of knowing, for instance, how the mathematical models hook up with the physical world, and how to claim that structural explanations are genuine accounts of physical phenomena. And for us this is a serious drawback, since we take structural explanations to be genuine explanations of physical phenomena.

In the following, final section, we will argue that the step from Suárez's inferentialism to a more substantive account of representation, however, is not too long. In particular, Suárez allows for additional, contextual necessary conditions that a scientific representation must satisfy, but denies that these conditions can contribute to the objectivity of representation, which for him is a non-contextual notion. On the contrary, we will now see how insisting on the pragmatic aims of the users of the model can solve a series of difficulties of the isomorphic account of representation, so as to achieve that genuine informativity of the model about the world that Suarez was striving to capture. This will also entail a rapprochement between the two allegedly opposed camps that we have presented in this section.

\section{Minkowski’s model and structural explanations}

In a nutshell, our argument so far can be summarized as follows: (i) the relativistic contractions and dilations need to be explained; (ii) structural explanations provide a genuine explanation of these physical phenomena; (iii) structural explanations are not to be cashed out in terms of causal or mechanical or DN type of explanations; (iv) it then becomes highly plausible that such explanations require some form of morphism between the model and the target, so that the semantic view, supplemented with a robust doses of pragmatism, seems the only explanation for their genuine explanatory character. Note that the first three premises have been argued for in the first section. In order to defend the conclusion (iv), we will begin from the same starting point that is considered central by the proponents of the pragmatic view, namely that of giving a more precise account of the way cognitive agents use scientific models.

Going back to our case study, we can introduce the problem of explaining structural explanations in these terms: which contextual, necessary conditions should a mathematical model meet in order to serve as a provider of a structural explanation? While we will keep on considering 
structural explanations in Minkowski spacetime as our case study, we will leave open the question to which extent the remarks offered in this section can be suitably generalized.

First, we have seen that a structural explanation typically does not require any specific ontological commitment about what is represented by the structure, i.e., in our case it is neutral with respect to the traditional division between substantivalism and relationism about Minkowski spacetime. ${ }^{10}$ It follows that a cognitive agent can use models of Minkowski spacetime to provide a consistent structural explanation, without being committed to a specific ontology of the controversial type illustrated by Brown (i.e., a causal form of substantivalism)

Second, in a structural explanation the physical explanandum $B$ is understood in terms of the relational properties of its formal counterpart $A$. Remember that structural explanations make essential use of the mathematical laws and principles defining the model. Of course, such laws and principles ought to codify physical postulates of the theory: in our case, of the main characteristics of the mathematical model is the invariant quantity $\Delta \mathrm{S}$, which represents the speed of light in all inertial frames:

$$
\Delta S=\sqrt{\left(t_{p}-t_{q}\right)^{2}-\left(x_{p}-x_{q}\right)^{2}-\left(y_{p}-y_{q}\right)^{2}-\left(z_{p}-z_{q}\right)^{2}}
$$

The main point is, however, that in order to transfer knowledge about the relational properties of the model $A$ into knowledge about the relational properties of the physical explanandum $B$, we must assume that the relational properties and laws exemplified by the model $A$ are also (at least in part) relational properties and laws exemplified by $B$. Without this exemplification of the relations and laws of $A$ by $B$, no transfer of knowledge from $A$ to $B$ could ever occur, and any inference drawn in the model would not be about the physical world. As a consequence, objectivity would be lost. In other words, the performance on the part of an agent of a structural explanation of a physical phenomenon presupposes the assumption of a partial isomorphism (French and Ladyman 1999) between the relations exemplified by the model $A$ and those exemplified by the target $B$.

However, how can an isomorphism exist between an abstract and a concrete structure? In order to solve this difficulty, imagine a concrete sphere designed on a board with chalk: this has certain structural features and properties that we can determine by studying spheres in an abstract and idealized manner (for instance, we imagine that any point of the surface is really equidistant from the center). what matters for us is that it is only if the Earth and the sphere on the board exemplify approximately and partially the same structure of the abstract sphere that we can suitably transfer knowledge from the latter to the two former, concrete, physical objects. It is in virtue of such an assumption of partial isomorphic correspondence that structural explanations are

\footnotetext{
${ }^{10}$ Whether it is also neutral toward a form of spacetime structural realism is of course much less clear. But see below.
} 
considered effective. The correspondence is partial because the Earth is not exactly spherical, of course, but depending on our cognitive aims, we can decide to go ahead with the given approximation and treat the Earth as if it were a perfect sphere.

Going back to questions debated in 2, it is in virtue of an assumption of partial isomorphism of this kind that the Minkowskian model can be considered informative about the physical world, and therefore, whenever the right contextual situations obtain, enable informed users to give a genuine structural explanation of a physical phenomenon. Furthermore, notice that it is the use that the speaker does of a certain model that solves many of the problems of the isomorphic account of scientific representation. First of all, it is the fact that we intentionally use a model to represent something physical that makes the relation of isomorphism, which is per se symmetric, asymmetric. By using $\mathrm{x}$ to represent $\mathrm{y}$, we thereby automatically select one of the two directions of correspondence of model and world. Secondly, insisting on the particular interests of the user, one can obviously avoid all the underdetermination claims, since one uses particular aspects of the model for particular purposes, selecting particular aspects of reality as it is more convenient for the aim at hand. Thirdly, as we have seen, also the problem of correspondence between an abstract and a concrete structure is overcome: the ante rem abstract structure (the abstract sphere) is exemplified by the concrete physical system, in such a way that the former partially exists in re in the latter (the concrete sphere on the board).

This remark is linked to another important point concerning the reality of Lorentz contractions, with which we opened the paper. If conceived as structural explanations, the geometrical explanations provided by SR do not conceive of relativistic phenomena like Lorentz contraction as merely perspectival, in the sense of unreal. As already argued in 1, Lorentz contraction should be conceived as real in the same sense in which the structure of spacetime is real. Minkowski spacetime is real to the extent that it is genuinely exemplified by physical fields and events. Likewise, Lorentz's contractions are real to the extent that they are exemplified by physical systems in reciprocal motion, as illustrated by a geometrical explanation of the relevant phenomena. In order for this conception of relativistic phenomena to be justified, however, one necessarily has to acknowledge that Minkowski spacetime models, and in particular the invariance of the spacetime interval, actually captures a feature of reality, in the sense that the latter is a concrete instance of the former. The difference between a mere mathematical explanation and a mathematical explanation of a physical phenomenon is therefore given by the existence of an exemplification relation: without the assumption of this exemplification relation between the target and the model, the geometrical accounts provided by SR would end up being a mere logico/mathematical derivation of the 
explanandum - something more similar to a Deductive Nomological explanation, realized thanks to a mere mathematical law belonging to a mere mathematical structure. ${ }^{11}$

Of course, by insisting on the importance of structural explanations in certain contexts, we are not denying that, in other contexts, causal explanations may not be more appropriate. It is not necessary for our main claim to argue that the isomorphic approach works in all possible contexts, but only that it works in our particular case study, i.e., as an explanation of structural explanations in SR. ${ }^{12}$ On the contrary, as already anticipated above, we accept a contextual/pragmatic dimension of explanation and therefore of the use of models. And we believe that there is no general set of necessary and sufficient conditions that in all possible contexts can tell us where a causal rather than a structural explanation is appropriate. On the contrary, it is not impossible that the two types of explanation can coexist even in the same theory. Obviously, depending on the kind of allowed surrogative reasoning (causal explanation vs. structural explanation), one imposes different conditions for rationally believing in the informativity of the representation.

\section{Conclusion}

We would like to end this paper with a general consideration about the theory of scientific representation. The point of departure of Suárez's defence of the inferentialist view vis à vis the semantic view was that only the former accounts for the way cognitive agents use models in science. But it is not clear at all why defenders of the isomorphic approach could not make room for a decisive pragmatic component of scientific representation (see Debs and Redhead 2007). Consider the question: what justifies a rational agent to interpret the product of her surrogative reasoning as a piece of knowledge about the target? Since knowing p entails $\mathrm{p}$, we must assume that there is some sort of truthlikeness in the representation allowed by a model. Clearly, if the fact that the model enables us to draw correct inferences about the target is objective in more than an epistemic sense, this fact must receive an explanation in terms of the existence of some objective relationship between the model and the world. We have argued that what kind of relationship holds is contextual (in the context of the geometrical/structural explanations it is the assumption of a partial isomorphism that needs to be assumed), but this contextuality does not go against a genuine 'objectivity' (in the stronger sense) of the resulting scientific explanation, because some kind of grasp into physical reality is still always necessary in order to account for the rationality of any kind

\footnotetext{
${ }^{11}$ One can recognize this charge against the effectiveness of geometrical explanations also in Brown's works (see Felline 2010).

${ }^{12}$ For problems with the isomorphic approach, see Batterman (2010).
} 
of surrogative reasoning. This conclusion leaves space to the idea that the two accounts of representation end up being much closer than their defenders tend to admit. Possibly, they are the two inseparable sides of the same coin.

\section{Acknowledgments}

The authors thank Vesselin Petkov for his careful reading of a previous version of this paper, which eliminated a mistake. Laura Felline's research is founded by a Master and Back scholarship from Regione Sardegna. She would also like to thank all the members of Alophis - Applied Logic and Philosophy of Science - group for daily support and help.

\section{References}

Balashov, Y., Janssen, M. (2003), "Presentism and Relativity" The British Journal for the Philosophy of Science 2003 54(2): 327-346.

Batterman, R., (2002), The Devil in the Details: Asymptotic Reasoning in Explanation, Reduction and Emergence. Oxford: Oxford University Press. , (2010), "On the Explanatory Role of Mathematics in Empirical Science" The British Journal for the Philosophy of Science 61: 1-25;

Brown, H. (2005), Physical Relativity. Spacetime Structure from a dynamical perspective. Oxford University Press, Oxford. and Pooley O. (2006), "Minkowski Spacetime: a Glorious Non-Entity", in Dieks D. (ed.) The Ontology of Spacetime, Elsevier, pp. 67-89.

and Timson, "Why special relativity should not be a template for a fundamental reformulation of quantum mechanics", In Physical Theory and its Interpretation: Essays in Honor of Jeffrey Bub, ed. William Demopoulos and Itamar Pitowsky, 29-42. Springer. Clifton, R., (1998), "Structural Explanation in Quantum Theory" Eprint: http://philsci-archive.pitt.edu/archive/00000091/00/explanation-in-QT.pdf, unpublished.

Debs T. Redhead M. (2007), Objectivity, Invariance and Conventions: Symmetries in Physical Science, Harvard University Press, Harvard University Press.

Dorato, M. (2006), "The irrelevance of the presentism/eternalism debate for the ontology of Minkowski spacetime", in D. Dieks (ed.), The Ontology of Spacetime, Elsevier, pp. 93-109.

Dorato, M. (2007), "Relativity Theory between Structural and Dynamical Explanations", International Studies in the Philosophy of Science, Volume 21, Issue 1 March 200795 - 102

Dorato M. and Felline L. (forthcoming), Scientific Explanation and Scientific Structuralism, in Bokulich A., Bokulich P. (eds.), Scientific Structuralism, Boston Studies in Philosophy of Science,

Einstein A. (1919), "What is the Theory of Relativity?", Times (London). 28 November 1919. Reprinted in Einstein (1982, 227-32) Ideas and Opinions, Crown Publishers, Inc., New York Felline L. (forthcoming), "Scientific Explanation between Principle and Constructive Theories" 
Feynman R.,Leighton, R. and Sands, M. (1963), The Feynman Lectures on Physics, Addison Wesley, Redwood City, California.

French, S., (1999) 'Models and Mathematics in Physics: The Role of Group Theory', in From Physics to Philosophy, ed. J. Butterfield and C. Pagonis (Cambridge: Cambridge University Press), pp.187-207.

and Ladyman, J. (1999) 'Reinflating the Semantic Approach', International Studies in the Philosophy of Science 13, 103-121.

Frigg, R., (2006), "Scientific Representation and the Semantic View of Theories", Theoria 55: 37 53. http://philsci-archive.pitt.edu/archive/00002926/01/Scientific_Representation.pdf.

Giere, R., (1988) Explaining Science. A Cognitive Approach. Chicago. University of Chicago Press. Hartmann, S., (1999), "Models and stories in hadron physics." in Models as Mediators. (Eds) Morrison, M. and Morgan, M.. Cambridge: Cambridge University Press. 326-346. Eprint: http://philsci-archive.pitt.edu/archive/00002433/01/Stories.pdf

Hughes, R.I.G. (1989a), "Bell's theorem, ideology, and structural explanation", in Cushing, J. and McMullin, J. (eds.), Philosophical Consequences of Quantum Theory, Notre Dame, pp. 195-207.

(1989b), The Structure and Interpretation of Quantum Mechanics, Harvard University Press.

(1993), Theoretical Explanation, Midwest Studies in Philosophy XVIII: 132-153.

Minkowski, H. (1908) "Space and Time," as reprinted and translated in V. Petkov (ed.), Minkowski Spacetime: A Hundred Years Later (Springer, Berlin, Heidelberg, New York) pp. xiv-xlii.

Norton J.D. (2008) "Why constructive relativity fails", British Journal for the Philosophy of Science, 59(4): 821-834;

Petkov V. (2007) "Relativity, Dimensionality, and Existence". In: V. Petkov (ed.), Relativity and the Dimensionality of the World, Springer, Berlin, Heidelberg, New York pp. 115135.

(2009) Relativity and the Nature of Spacetime, 2nd ed. (Springer, Berlin, Heidelberg, New York)

Sider, T. (2003), Fourdimensionalism. An ontology of persistence and time. Clarendon Press.

Suárez, M. (1999), "Theories, Models, and Representations", in L. Magnani, N. J. Nersessian and P. Thagard (eds.), Model-Based Reasoning in Scientific Discovery. New York: Kluwer, 75-83. (2003), "Scientific Representation: Against Similarity and Isomorphism", International Studies in the Philosophy of Science 17: 225-244.

(2004), "An Inferential Conception of Scientific Representation", Philosophy of Science (Symposia), 71: 767-779.

Suppe, F. (1977). "The search for Philosophic Understanding of Scientific Theories." In The Structure of Scientific Theories, ed. F. Suppe, pp. 3-232. Urbana: University of Illinois Press.

Suppes, P. (1962) Models of data. In E. Nagel, P. Suppes, \& A. Tarski (Eds.), Logic, Methodology and Philosophy of Science: Proceedings of the 1960 International Congress. Stanford: Stanford University Press, 252-261.

(1967). "What is a Scientific Theory?" In: Philosophy of Science Today. (Morgenbesser S, ed), 55-67. New York: Basic Books

van Fraassen, Bas C. (1980), The Scientific Image. Oxford: Oxford University Press.

,(2008) Scientific Representation: Paradoxes of Perspective. Oxford: Oxford University Press 